Baltic Astronomy, vol.4, 510-518, 1995.

\title{
A PORTABLE GPS TIME SOURCE FOR WET USE
}

\author{
D. J. Sullivan
}

Department of Physics, Victoria University of Wellington, P.O. Box 600, Wellington, New Zealand

Received September 1, 1995.

\begin{abstract}
An overview of the Global Positioning System is presented with an emphasis on its use as a convenient and accurate time source. An outline of a portable GPS time source for use in the Whole Earth Telescope high-speed photometry is given.
\end{abstract}

Key words: instrumentation: time sources, photometers - time space vehicles

\section{Introduction}

The pulsating white dwarfs exhibit frequencies in their light curves with periods as small as $\sim 100 \mathrm{~s}$. The asteroseismological study of these objects using the Whole Earth Telescope (WET) network demands local clock accuracy of better than about a second, if time synchronization errors are not to degrade the quality of the temporal spectroscopy that is produced. Given the possible accuracy of modern clocks, this requirement may seem trivial. The reality is, that unless particular care is taken, it is all too easy to be "out by seconds", even in the age of ever-expanding electronics and computers.

The WET observer community has developed a considerable expertise in the art of precise high-speed photometry, and there is no doubt a consensus view that we would be very unwise to rely on the proverbial observatory "people's photometer" for this work. Given the grand tradition of astronomers as the first time keepers, it is rather ironic that one can argue - with some justification - that we shouldn't rely on the observatory clock either!

We do not have to! There now exists a convenient, accurate and highly reliable time source for observers anywhere on the Earth. This is the Global Positioning System (GPS) developed by the US 
Department of Defence for the accurate ( $\sim$ meters) positioning and deployment of military forces. Timing information from the atomic clocks, that are an integral part of GPS, is readily available to any user equipped with a special, relatively inexpensive ( US\$600) receiver.

I have been using GPS for about a year now to maintain synchronism of my local clock for accurate time series work. I have recently started work on a stand-alone GPS timing unit that can also be incorporated into the evolving WET instrumentation package that already includes the counting hardware designed by Chris Clemens and the PC software developed by Ed Nather.

The purpose of this paper is to briefly explain GPS, give an overview of the project and provide a summary of the current state of the design process.

\section{The Global Positioning System}

GPS uses highly accurate clocks in a network of satellites orbiting the earth to enable the determination of user positions using time delay measurements. There are actually four atomic clocks (two cesium and two rubidium) on each satellite.

The complete system consists of a constellation of satellites, ground control and monitoring stations and (for a particular user) a (now) relatively inexpensive receiver/microprocessor. This receiver accepts information transmitted by the satellites and computes the position of the user to $\sim 1$ meter (for authorized military users) and $\sim 100$ meters for others. This is achieved by measuring the signal pro pagation times from an appropriate number of satellites, whose positions are accurately known. Each satellite transmits signals (carrier frequencies $\sim \mathrm{GHz}$ ) that contain information on satellite availability (an almanac), allow precise computation of the satellite position (an ephemeris) and permit measurement of the signal propagation time between it and the receiver.

The military specifications for this system required the line-ofsight 24-hour availability of a minimum of four satellites (see below) from all positions on the Earth. This has been achieved with the deployment of 24 satellites, arranged in 6 different orbital planes, with each inclined at an angle of $55^{\circ}$ to the equatorial plane. All satellites are in nearly circular orbits at an altitude of about $20200 \mathrm{~km}$ above the Earth, with a corresponding orbital period of 12 sidereal hours. 
The signals from three satellites (three "intersecting spheres" geometry) are sufficient to uniquely determine the three-dimensional position of the user, if the receiver's clock is accurately synchronized with the clocks in the satellites. The $1 \mathrm{~m}$ distance precision requirement translates into a 3 nanosecond clock requirement, which can only be satisfied in isolation by an atomic clock. Quartz clocks are not good enough.

One of the really smart design features of the GPS system is that it has obviated the need for an expensive receiver clock by utilizing the signal from an additional satellite to perform the clock synchronization. Atomic clocks, although relatively portable, are still somewhat cumbersome and expensive. Hence, in practice, the signals from four satellites are used to determine the 3D position and synchronize the local (crystal) clock. Only three satellites in view are necessary to synchronize the local clock and compute the receiver's two-dimensional position relative to an assumed mean Earth radius, while only one satellite signal is necessary to synchronize the receiver clock if it is at a known position - such as an observatory (presumably!).

Incidentally, the civilian use of GPS, without utilizing special techniques (such as differential operation) is normally limited to positioning accuracy of $\sim 100$ meters, as a result of deliberate degradation of the information quality available in the signals ("dithering"). However, for the time synchronization use discussed here, this is irrelevant, as this position uncertainty still translates into a time synchronization accuracy of better than a microsecond!

\section{The GPS receiver}

The rapidly expanding civilian GPS market has led to the availability of a variety of receivers. Most of these units focus on the positioning capability, and do not electronically pass the accurate time information to the user. Magellan Systems Corporation (960 Overland Court, San Dimas, California, U.S.A.) market an OEM board-level unit that costs about US $\$ 600$ when purchased along with an active antenna. The board includes high frequency analogue and digital electronics, a microprocessor unit and suitable software to accomplish the necessary tasks. The small antenna unit (size $\sim 10 \mathrm{~cm}$ ) will function over $30 \mathrm{~m}$ lengths of standard coaxial cable.

This is the unit I have been using for time synchronization. In addition to position data and other information on GPS performance 
provided in ASCII strings via a serial interface port, the unit provides both $1 \mathrm{~Hz}$ and $1 \mathrm{kHz}$ pulses synchronized to either GPS time or UTC.

There is actually a lot going on in a GPS receiver, and its convenient availability is a direct result of the modern miracle of microelectronics - analogue and particularly digital. The apparently simple receiver is actually a (very) "smart box" and it hosts a computationally intensive operation.

A Magellan 5 channel unit in full operation has separate channels determining signal propagation times from the current four optimally placed satellites, while a fifth channel is searching for the next best satellite (using data from the initially downloaded satellite constellation almanac). The four measured propagation times incorporating local clock error ("pseudoranges") are used to iteratively solve four nonlinear equations in order to obtain the four unknowns: clock error and three position coordinates. These calculations in turn rely on knowledge of the accurate positions of the active satellites, provided by the regularly downloaded ephemerides in the form of current satellite orbital parameters. Interestingly, no attempt is made to maintain synchronization of the satellite clocks, as atomic units perform optimally when left to run freely. Instead the individual offsets and the overall GPS-UTC difference are determined by regular measurements at the ground control and monitor stations, and this information is incorporated in each periodically updated satellite ephemeris.

A convenient feature of the Magellan unit for timing purposes is the hierarchy of operating levels, ranging from full operation (providing $3 \mathrm{D}$ position and local clock synchronization) to minimal operation, whereby only one satellite signal and the last determined (or user-supplied) position are used to synchronize the local clock.

\section{The VUW fast photometer}

Fig. 1 provides a schematic overview of the VUW fast photometer, including the procedure used for accurate time keeping. A useful astronomical clock requires a quality frequency source, a counting mechanism and a procedure for checking/maintaining synchronism with UTC. Currently in New Zealand, I maintain a stand-alone clock in synchronism with international broadcast time and use appropriate signals from this unit to gate the logic in a special counter/buffer interface to a micro/PDP-11 computer (van der Peet 1987). The control program ("tsnew") in the PDP-11, functioning in priority 
interrupt mode, keeps track of time by counting appropriate (usually $1 \mathrm{~Hz}$ ) clock pulses from the external unit. A $0.1 \mathrm{~Hz}$ signal from the clock to the computer on the even 10 second rollover is initially used to synchronize the clock counting code, in order to eliminate the possibility of the computer clock being in error by an integral number of seconds from the displayed clock time (the local standard).

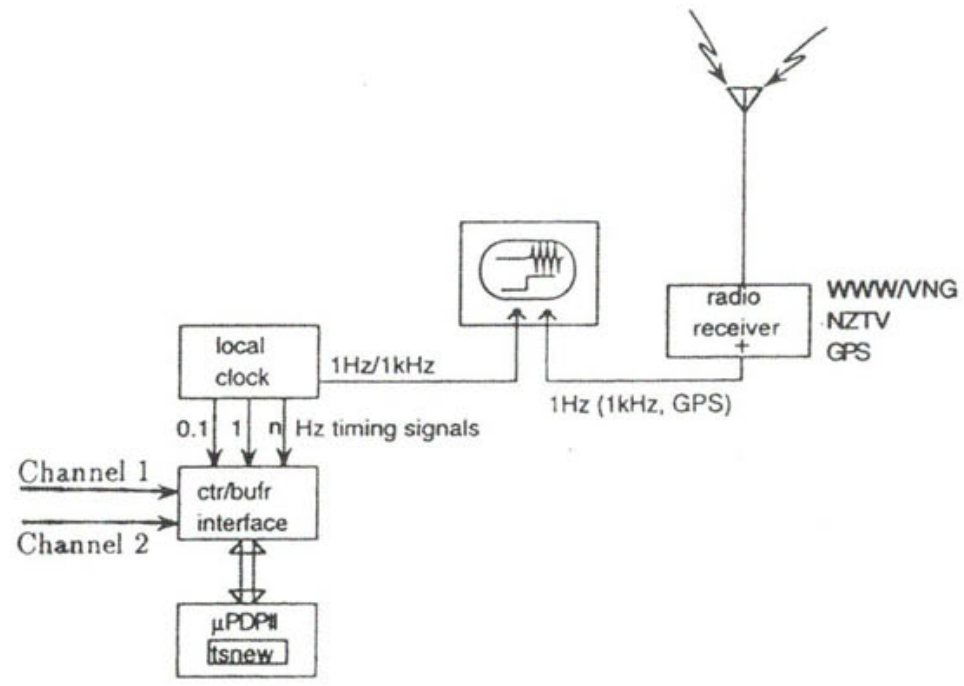

Fig. 1. The current VUW fast photometer configuration and the method used to maintain time synchronization.

My New Zealand observing base is Mount John University Observatory (MJUO), which is a field station of the University of Canterbury, Christchurch. For all my years as a guest observer there, I have had to provide my own time standard, if for no other reason than the fact that no Canterbury astronomer is, or has been, interested in accurate time work. In simple terms, there is no observatory clock! Motivated by my early interest in timing lunar occultations, where millisecond absolute accuracy was deemed useful, I and a collaborator (Sullivan and Trodahl 1980) developed a portable time standard, based around a commercial temperature-controlled quartz oscillator. Battery back-up kept the crystal continuously operating in order to achieve optimum stability, while suitable digital electronics for adding or subtracting pulses fed to the chain of counters, enabled the displayed time to be kept (digitally) close to synchronism with UTC. The best stability achieved by the quartz clock is 
about 1 part in $10^{8}$ (better than a millisecond per day). Suitable signals available at MJUO for maintaining time synchronism with UTC are WWV (Hawaii) and VNG (Australia), with the latter being somewhat unreliable for operational reasons at the source.

Without doubt, the most effective way to monitor and adjust the local clock is the method depicted in Fig. 1. A dual trace oscilloscope, triggered by the steady digital signal (usually $1 \mathrm{~Hz}$ ) from the local clock, is used to "simultaneously" view (chopping mode) both the clock signal and the external timing signal. The integrating properties of the eye combined with the persistence of the screen phosphor allow even low-level noisy signals to be used to check clock alignment. Given signal propagation times of about $10 \mathrm{msec}$ (Australia) and $30 \mathrm{msec}$ (Hawaii), local clock accuracy of about a millisecond is achievable. However, signal strengths have always been a problem, so I sought alternatives. One such alternative uses the frame synchronization signal $(50 \mathrm{~Hz})$ from a local TV channel, which is controlled by a rubidium oscillator. As this frequency is derived from a rubidium atomic standard, only one absolute "fix" with either VNG or WWV can be followed by regular comparisons of the local clock $50 \mathrm{~Hz}$ with the extremely stable TV signal, thus obviating the need to regularly receive broadcast time.

However, the situation changed radically with the introduction of a GPS time source (early 1994). The $1 \mathrm{~Hz}$ and $1 \mathrm{kHz}$ signals from the Magellan unit enable setting of the local clock to $\sim \mu$ sec at any time, using the oscilloscope method depicted in Fig. 1.

\section{A portable WET GPS unit}

Although the timing pulses output by the Magellan GPS units are simple enough, the other information is presented in "userunfriendly" ASCII strings. Being an OEM development unit, it is meant to be incorporated into another system. My current working configuration interacts with a laptop running a developmental display program supplied by Magellan. With a bit of "know-how" the system is perfectly satisfactory for time synchronization.

However, in contemplating how I would build the Magellan board into a stand-alone timing unit that also would supply some of the other items of interest to observers (such as position, unit functionality, etc), it became clear to me that I should also ensure compatibility with the existing WET instrumentation. Although my existing instrumentation is more than adequate for fast photometry, 
and it is nicely tailored for use on the MJUO $1 \mathrm{~m}$ telescope, computer replacement time is fast approaching. The computer unit is more than a decade old, so maintenance is becoming an issue, and the hardware is "luggable" rather than portable. I am therefore steadily migrating towards use of the Clemens/Nather WET equipment. Adding a GPS timing capability to this instrumentation, it seems to me, would improve an already very nice package. Suitable use of a GPS unit would virtually eliminate timing errors during use of this system.

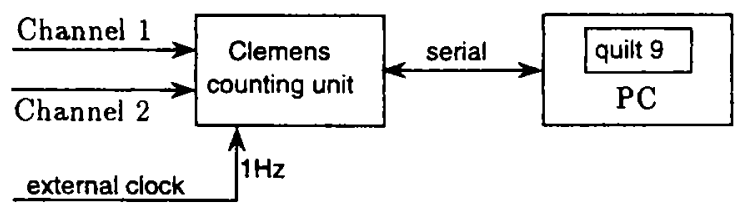

Fig. 2. A simple schematic of the current Clemens/Nather WET instrumentation.

Fig. 2 provides a simple overview of the existing Clemens/Nather equipment. The microprocessor-controlled multiple counter/buffer unit has an internal crystal frequency standard that is used to control counter gating (and therefore set photon integration intervals). This internal frequency can be synchronized with UTC with an external $1 \mathrm{~Hz}$ signal from an observatory clock. The counter unit communicates with an IBM-compatible PC, running Ed Nather's control program "Quilt9", via the standard serial port. The PC program keeps track of time by using the receipt of a 9-byte data string every second - as a $1 \mathrm{~Hz}$ clock signal. Although this scheme works in a satisfactory manner, it can be improved, without changing the basic methodology, by adding a suitable GPS unit.

Fig. 3 provides a schematic view of the proposed WET data acquisition system, with the added GPS capability. An important design criterion is that the new configuration be "downwards compatible". Namely, remove the new GPS unit and the system will function essentially as before.

Under the control of a Z8 microprocessor, the GPS box will display useful information (e.g. time and position) directly to the observer as well as monitor the serial "traffic" between the counting unit and the PC, now running a modified control program ("Quilt9++" - thanks Ed!). The accurate $1 \mathrm{~Hz}$ UTC signal from the GPS unit 


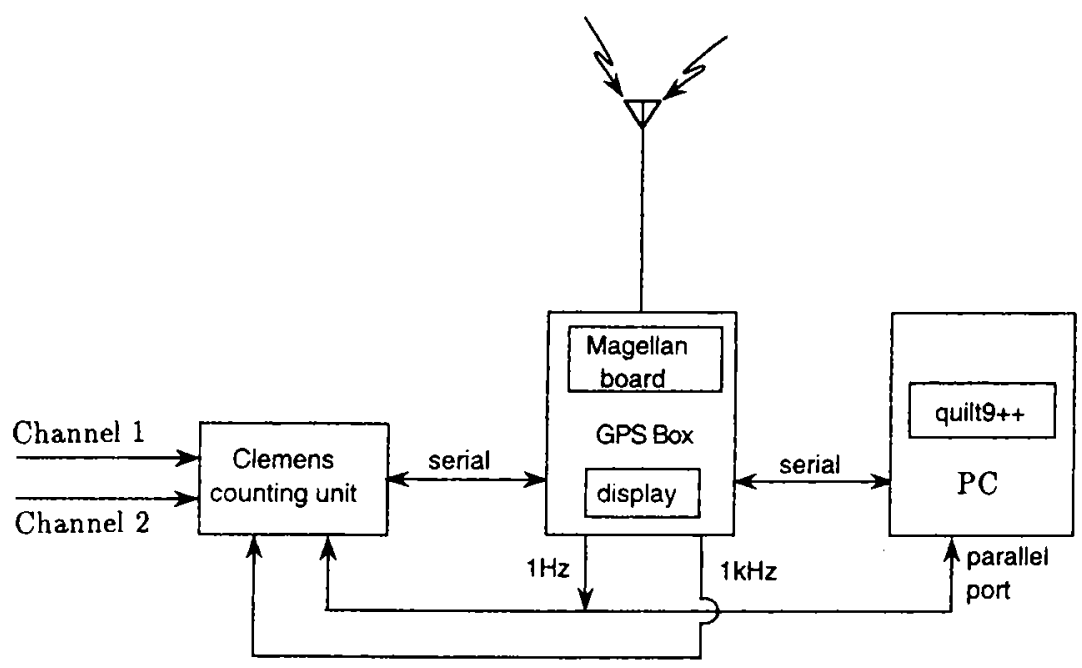

Fig. 3. A schematic block diagram illustrating the proposed addition of a GPS timing capability to the standard WET data acquisition equipment.

will be used to synchronize the counter integrations and provide time directly to the PC (via one pin of the standard parallel port, as this is readily available on all PCs). The GPS $1 \mathrm{kHz}$ signal will also be available to the counter unit as an alternative/replacement higher frequency standard. By monitoring the PC serial port, the GPS box will be able to provide on demand (e.g. at program initialization time), serial strings providing information on such things as GPS functionality, current UTC time and position coordinates. Such a scheme should eliminate a number of sources of error in the current WET observing network.

\section{Conclusions}

GPS is a fascinating system with many intricacies, and in the interests of brevity I have glossed over many of the details (e.g. Hofmann-Wellenhof 1990). As with many modern instruments, the user can remain largely ignorant of these details. However, I believe that the intelligent astronomical user of GPS should at least have a rough working knowledge of its operation. It is my view that the GPS timing unit proposed here, when combined with the existing Clemens/Nather WET photometer instrumentation, will remove any uncertainties in timing from the overall WET operation. 
Acknowledgments. It first occurred to me to use GPS as a time source when I attended a conference on precision photometry at the South African Astronomical Observatory in February 1993. The Electronics Workshop personnel there had just taken delivery of an OEM GPS receiver for development purposes. I am grateful for their focussing of my mind on this topic. It is a pleasure to acknowledge help from discussions with Ed Nather and Chris Clemens, and thanks are also due to the VUW (Physics) Electronics workshop personnel (Bob Halford and Grant Parratt) for providing assistance in the development work for the GPS timing unit.

\section{References}

Hofmann-Wellenhof B., Lichtenegger H., Collins J. 1990, Global Positioning System - Theory and Practice, Springer-Verlag, Berlin

Sullivan D.J., Trodahl H.J. 1980, Victoria University of Wellington, (unpublished)

van der Peet A.J. 1987, M. Sc. Thesis, Victoria University of Wellington 